\title{
DYNAMICS OF LIGHT-ABSORPTION VARIATIONS INDUCED IN A BISMUTH SILICATE CRYSTAL BY VISIBLE LASER ILLUMINATION
}

\author{
E. S. Khudyakova, ${ }^{1 *}$ M. G. Kisteneva, ${ }^{1}$ \\ S. M. Shandarov, ${ }^{1}$ T. A. Kornienko, ${ }^{2}$ A. L. Tolstik, ${ }^{2}$ \\ and Yu. F. Kargin ${ }^{3}$
}

UDC 535.343.2

\begin{abstract}
We present the results of experimental studies of the dynamics of the photoinduced optical absorption in a bismuth silicate crystal subject to continuous laser irradiation with wavelengths of 532 and $655 \mathrm{~nm}$. The semiconductor-laser light beam with the wavelength $\lambda=655 \mathrm{~nm}$ causes the crystal bleaching at this wavelength, whereas its exposure to a shorter-wavelength irradiation from the optical-spectrum green region with a wavelength of $532 \mathrm{~nm}$ increases the optical absorption at both wavelengths, of 532 and $655 \mathrm{~nm}$. The experimental results are interpreted using the theoretical model which assumes that the crystal has deep defect centers of two types so that an electron at each of these centers can be in one of the states characterized by different photoionization cross sections.
\end{abstract}

\section{INTRODUCTION}

The sillenite-type $\mathrm{Bi}_{12} \mathrm{MO}_{20}$ crystals, where $\mathrm{M}=\mathrm{Si}$, Ge, and $\mathrm{Ti}$, are widely used as photorefractive materials for dynamic holography $[1,2]$. The photoinduced optical-absorption variations inherent in these crystals, i.e., the photochromic effect, are related to the complicated energy structure of their defect centers $[1,3,4]$, which in turn affects both the dynamics of formation of the photorefractive holograms and the photorefractive sensitivity. The photoinduced distribution of the charge carriers (electrons) over the defect centers, which is accompanied by the photochromic effect, can be one of the mechanisms of control of the above-mentioned parameters. For example, if the bismuth titanate crystals are irradiated from the visible range of the electromagnetic-radiation spectrum, then not only the photoinduced variations occur in the spectrum of their optical absorption [4], but also the photorefractive sensitivity in the near infrared (IR) region increases $[1,5]$. In this respect, the development of the quantitative models which describe the photoinduced effects in the sillenite crystals, is of interest for realizing the dynamic-holography effects in the long-wavelength region of the visible spectrum and the near-infrared region.

In this work, we present the results of the studies of the dynamics of the photoinduced optical absorption, which is observed in the bismuth silicate crystal $\mathrm{Bi}_{12} \mathrm{SiO}_{20}$ when it is exposed to continuouswave laser irradiation with wavelengths of 532 and $655 \mathrm{~nm}$.

\section{EXPERIMENTAL FACILITY AND TECHNIQUES}

The experimental setup for studying the dynamics of photoinduced absorption, which uses two laser sources in an undoped $\mathrm{Bi}_{12} \mathrm{SiO}_{20}$ (BSO) crystal with section (100) and thickness $d=7.3 \mathrm{~mm}$, is shown in

* lenoliya@rambler.ru

1 Tomsk State University of Control Systems and Radioelectronics, Tomsk, Russia; ${ }^{2}$ University of Belarus, Minsk, Belorus; ${ }^{3}$ Institute of Metallurgy and Material Science of the Russian Academy of Sciences, Moscow, Russia. Translated from Izvestiya Vysshikh Uchebnykh Zavedenii, Radiofizika, Vol.57, No. 8-9, pp. 660-665, August-September 2014. Original article submitted November 11, 2013; accepted March 31, 2014. 


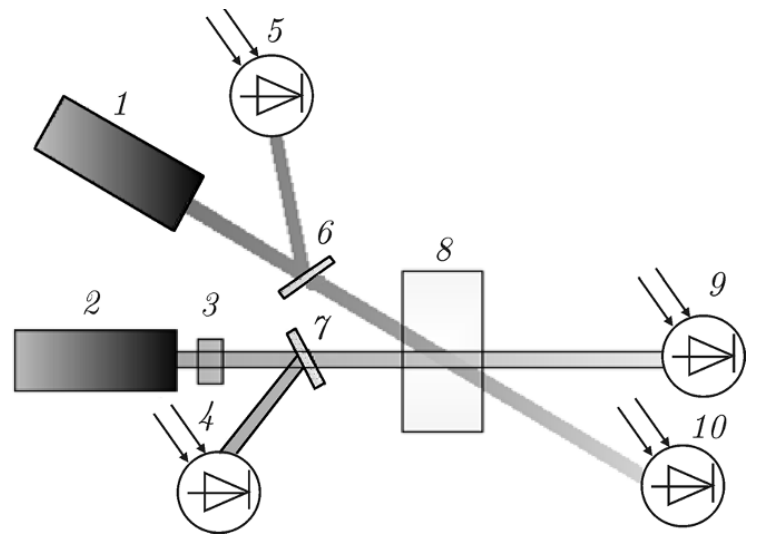

Fig. 1. Optical scheme for observing the dynamics of photoinduced variations in the absorption coefficient: semiconductor laser diode with an operating wavelength of $655 \mathrm{~nm}(1)$, continuouswave $\mathrm{Nd}$ :YAG laser with a radiation wavelength of $532 \mathrm{~nm}$ (2), collimator (3), beam-splitting plates ( 6 and 7$)$, BSO sample (8), and FD-24K photodiodes $(4,5,9$, and 10$)$. $S_{\mathrm{ph}}$. However, the time variation $\Delta k_{d}(t)$ in the absorption coefficient for each wavelength was calculated according to the relationship

$$
\Delta k_{d}(t)=\frac{1}{d} \ln \frac{I_{\mathrm{S}}(t) / I_{\mathrm{K}}(t)}{I_{\mathrm{S}}(0) / I_{\mathrm{K}}(0)}
$$

which rules out the influence of the differences in $S_{\mathrm{ph}}$ on the measurement results. In Eq. (1), $I_{\mathrm{S}}$ is the intensity of the radiation transmitting through the crystal, $I_{\mathrm{K}}$ is the radiation intensity for the check channel, and $d$ is the crystal thickness.

Two techniques were used in the experiments on the study of the absorption-coefficient dynamics in the BSO crystal. In the first technique, the photoinduced absorption variations were caused by a light beam with the wavelength $\lambda_{\mathrm{g}}=532 \mathrm{~nm}$, while the induced absorption variations were monitored by the sample transmission of radiation with another wavelength $\lambda_{\mathrm{r}}=655 \mathrm{~nm}$ using optical diode 10 . Optical diode 5 ensured control over the laser output power.

The second technique allowed us to study the dynamics of the natural photoinduced variations for a single wavelength. In this case, the crystal transmission was recorded using photodiodes 5 and 10 for radiation with the wavelength $\lambda_{\mathrm{r}}=655 \mathrm{~nm}$ and photodiodes 4 and 9 for the wavelength $\lambda_{\mathrm{g}}=532 \mathrm{~nm}$.

\section{EXPERIMENTAL RESULTS}

The dynamics of the absorption coefficient $\Delta k_{d}$ for the $\mathrm{Bi}_{12} \mathrm{SiO}_{20}$ crystal is shown in Fig. 2. The experiment started with irradiation of the crystal by the sounding laser radiation with the wavelength $\lambda_{\mathrm{r}}=655 \mathrm{~nm}$ at time $t_{0}=0$ after which its bleaching began to occur (Fig. 2, region 1 ). At $t_{1}=200 \mathrm{~s}$, the illumination radiation with a wavelength of $532 \mathrm{~nm}$ was switched on, which acted on the crystal up to the time $t_{2}=250 \mathrm{~s}$ and comparatively rapidly increased the optical absorption (Fig. 2, region 2). The subsequent experimental stages in regions 3, 5, and 7 are characterized by bleaching of the crystal, when it is affected only by the sounding radiation from the red region of the spectrum $\left(\lambda_{\mathrm{r}}=655 \mathrm{~nm}\right)$, and its darkening at this wavelength due to the effect of the radiation with $\lambda_{\mathrm{g}}=532 \mathrm{~nm}$ (Fig. 2, regions 4 and 6 ).

Figure 2 shows that the crystal bleaching $-\Delta k_{d}$, which is reached under the action of radiation of the red spectrum region, increases from $0.021 \mathrm{~cm}^{-1}$ in region 1 to $0.028 \mathrm{~cm}^{-1}$ in the last region 7 . At the 
Fig. 2. Dynamics of variations in the lightabsorption coefficient in the $\mathrm{Bi}_{12} \mathrm{SiO}_{20}$ crystal for a sounding beam with a wavelength of $655 \mathrm{~nm}$, which has an input flux density of $30 \mathrm{~mW} / \mathrm{cm}^{2}$ during the entire experiment $(t=0-1800 \mathrm{~s})$, with the switch-on of the laser irradiation with a wavelength of $532 \mathrm{~nm}$ and a flux density of $200 \mathrm{~mW} / \mathrm{cm}^{2}$ on the input edge of the crystal for the time intervals $t=200-250 \mathrm{~s}$ (region 2), 600-700 s (region 4), and 1250-1450 s (region 6). The circles and the solid curve denote the experimental data and the numerical-simulation results, respectively.

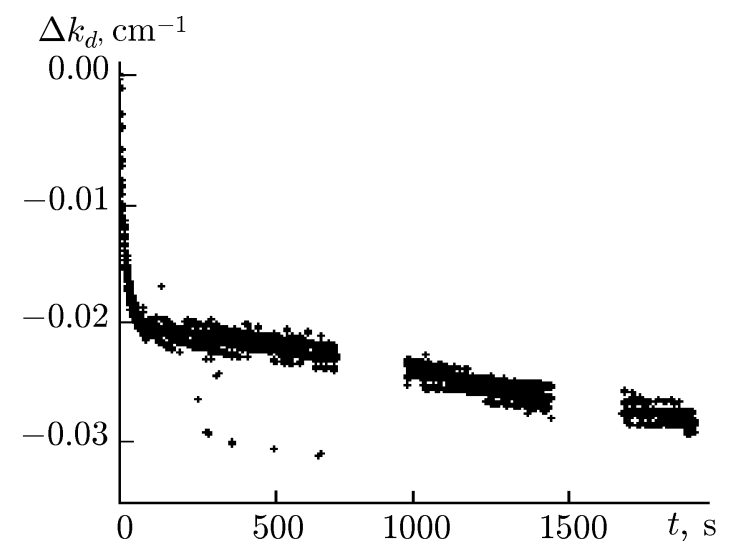

Fig. 3. Dynamics of variations in the lightabsorption coefficient for the fixed wavelength $\lambda_{\mathrm{r}}=655 \mathrm{~nm}$.
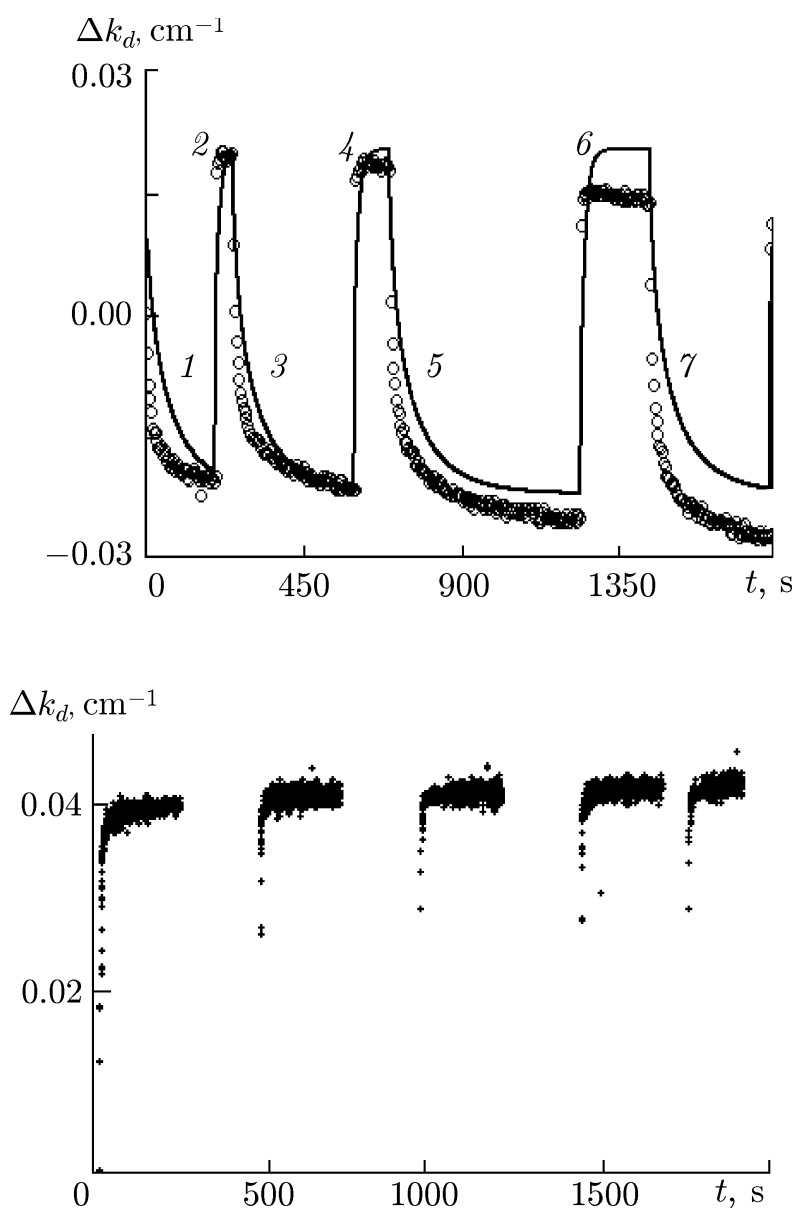

Fig. 4. Dynamics of variations in the lightabsorption coefficient for the fixed wavelength $\lambda_{\mathrm{g}}=532 \mathrm{~nm}$.

same time, the crystal darkening caused by the short-wavelength laser radiation decreases from 0.020 to $0.015 \mathrm{~cm}^{-1}$.

In the experiments on the study of the dynamics of the natural photoinduced absorption variations due only to light with the wavelength $\lambda_{\mathrm{r}}=655 \mathrm{~nm}$, radiation with flux density increased up to about $120 \mathrm{~mW} / \mathrm{cm}^{2}$ was used. The results of one of such experiments are depicted in Fig. 3 . It is seen in the figure that the absorption coefficient rapidly (for about $30 \mathrm{~s}$ ) decreases by a value of about $0.019 \mathrm{~cm}^{-1}$ after the crystal-irradiation onset at $t=0$. Then a slower crystal bleaching occurs and continues in the absence of irradiation, which was switched off at the times $t_{\text {off }}=720$ and $1440 \mathrm{~s}$ and again switched on at $t_{\mathrm{on}}=960$ and $1680 \mathrm{~s}$. During the entire experimental time $(1920 \mathrm{~s})$, the absorption-coefficient variations reached $-0.029 \mathrm{~cm}^{-1}$.

Figure 4 shows the dynamics of the natural variations in the absorption coefficient of the studied crystal $\mathrm{Bi}_{12} \mathrm{SiO}_{20}$ for green light with $\lambda_{\mathrm{g}}=532 \mathrm{~nm}$ and the input flux density about $200 \mathrm{~mW} / \mathrm{cm}^{2}$. It is seen in the figure that a photoinduced increase in optical absorption is observed for the above-mentioned wavelength. However, in contrast to the observed behavior of absorption at a wavelength of $655 \mathrm{~nm}$ (Fig. 3), the end of the crystal irradiation with $\lambda_{\mathrm{g}}=532 \mathrm{~nm}$ at the times $t_{\mathrm{off}}=240,720,1200,1680$ and $1920 \mathrm{~s}$ is accompanied by the dark relaxation of the induced variations by about $0.015 \mathrm{~cm}^{-1}$ for the time intervals about $250 \mathrm{~s}$, which last up to the next switch-on of the irradiating laser beam. As in the previous case, fast absorption variations at the initial region (its increase amounts to $0.037 \mathrm{~cm}^{-1}$ for $15 \mathrm{~s}$ ) are replaced by a comparatively slow absorption increase. 


\section{ANALYSIS AND DISCUSSION OF THE RESULTS}

The dynamics of the photoinduced light absorption in the $\mathrm{Bi}_{12} \mathrm{SiO}_{20}$ crystal is described by the model $[7,8]$ which assumes the presence of two types of the electrically neutral deep defect centers 1 and 2 such that an electron at each center can be in one of the states $D$ and $T$ characterized by the smaller and greater values of the photoionization cross section, respectively. According to the band diagram in Fig. 5, the first-type center (with the electron energies $D_{1}$ and $T_{1}$ ) is deeper than the second one (with the electron energies $D_{2}$ and $T_{2}$ ).

The probability of the electron localization in the states $D$ or $T$, each of which is characterized by its own potential well, is determined by the crystal temperature. Due to the electron-phonon interaction and the close spatial location of these two wells, the electron can tunnel through the separating barrier. It is assumed that some of the above-mentioned centers are ionized under the dark conditions and their charge is compensated by the radiation-insensitive acceptors with number density $N_{\mathrm{A}}$. Under the action of light, the electrons move from the nonionized centers to the conduction band and then recombine with the ionized deep centers to the states $D$ or $T$. Within the framework of this model, for the light with a wavelength of $532 \mathrm{~nm}$ whose energy quantum $\hbar \omega$ allows one to excite electrons to the conduction band from all centers $D_{1}, D_{2}, T_{1}$, and $T_{2}$, the absorption coefficient is determined by the expression

$$
\alpha_{\mathrm{G}}=\hbar \omega_{\mathrm{G}}\left(S_{1 D} N_{1}+S_{1 T} M_{1}+S_{2 D} N_{2}+S_{2 T} M_{2}\right),
$$

where $N_{1}$ and $N_{2}$ or $M_{1}$ and $M_{2}$ are the number densities of the nonionized centers at which the electrons are in the states $D_{1}$ and $D_{2}$ or $T_{1}$ and $T_{2}$, respectively, and $S_{1 D} S_{2 D}, S_{1 T}$, and $S_{2 T}$ are the photoionization cross sections corresponding to the above centers and states. For the light with a wavelength of $655 \mathrm{~nm}$, whose quanta are capable of ionizing only the centers $D_{2}$ and $T_{2}$, the absorption coefficient is determined by the expression

$$
\alpha_{\mathrm{R}}=\hbar \omega_{\mathrm{R}}\left(S_{2 D} N_{2}+S_{2 T} M_{2}\right) .
$$

Using the system of the rate equations [7] corresponding to this model, we numerically analyzed the dynamics of the observed effects for the experiment in which the absorption variations are induced by a light beam with the wavelength $\lambda_{\mathrm{g}}=532 \mathrm{~nm}$ and the absorption variations are tracked by the sample transmission of radiation with another wavelength $\lambda_{\mathrm{r}}=655 \mathrm{~nm}$. The numerical-simulation results are shown by the solid curve in Fig. 2 where the experimental data are also presented. The figure shows that our model developed in $[7,8]$ satisfactorily describes the dynamics of the development and relaxation of the photoinduced light absorption in the studied bismuth silicate crystal.

Figure 6 shows the numerical-simulation results for the dynamics of the number densities of the nonionized centers $N_{1}$ and $M_{1}$, as well as $N_{2}$ and $M_{2}$, subjected to the red and green laser irradiation.

If only the sounding red irradiation is switched on (regions 1, 3, 5, and 7), the electron number densities $N_{2}$ and $M_{2}$ in the states $D_{2}$ and $T_{2}$, respectively, decrease, while the electron number densities $N_{1}$ and $M_{1}$ at deeper centers in the states $D_{1}$ and $T_{1}$, respectively, increase. As a result, the crystal absorption for the illumination with a wavelength of $655 \mathrm{~nm}$ decreases. If irradiation with a wavelength of $532 \mathrm{~nm}$ (regions 2, 4, and 6) is switched on, then the electron number density $N_{1}$ in the state $D_{1}$ decreases, while the electron filling of the "shallow" centers in the states $D_{2}$ and $T_{2}$ increases. The electron number density at the center $T_{1}$ varies nonmonotonically. First, for approximately $15-20 \mathrm{~s}$ (curve $M_{1}$; regions 2,4 , and 6 ), 

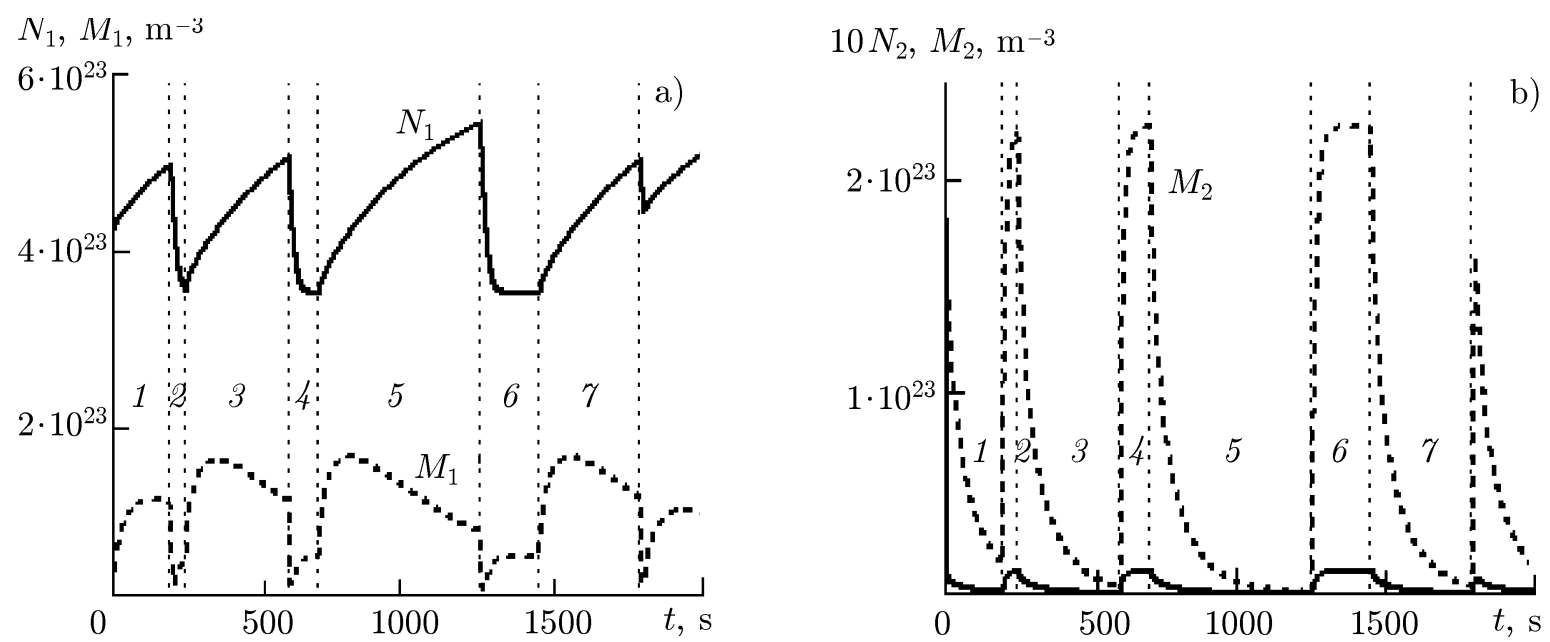

Fig. 6. Dynamics of the electron number density at the centers in the states $D$ and $T$ with small and large photoionization cross sections, respectively, for deeper centers $1(a)$ and shallower centers $2(b)$. The sounding beam with a wavelength of $655 \mathrm{~nm}$, which has a flux density of $30 \mathrm{~mW} / \mathrm{cm}^{2}$, is assumed to be constantly switched on $(t=0-2000 \mathrm{~s})$. The switch-on of the laser irradiation with a wavelength of $532 \mathrm{~nm}$ and a flux density of $200 \mathrm{~mW} / \mathrm{cm}^{2}$ in the crystal was simulated for the time intervals $t=200-250 \mathrm{~s}$ (region 2), 600-700 s (region 4), and 1250-1450 s (region 6).

irradiation with a wavelength of $532 \mathrm{~nm}$ leads to a decrease in $M_{1}$. Then the electron filling of the centers $T_{1}$ increases, which is related to the electron transitions from the state $T_{2}$ to the state $T_{1}$. This leads to an increase in optical absorption in the BSO crystal under irradiation with a wavelength of $532 \mathrm{~nm}$, which is experimentally observed for the two used wavelengths 655 and $532 \mathrm{~nm}$.

\section{CONCLUSIONS}

Thus, it has been established that during illumination of the undoped bismuth silicate crystal by laser radiation with wavelengths of 532 and $655 \mathrm{~nm}$, the reversible changes in its optical absorption are observed. They can influence the photorefractive characteristics of this crystal, which are important for realization of the dynamic-holography devices. It has been found out that the red irradiation bleaches the crystal, whereas its irradiation by the shorter-wavelength (e.g., green) radiation increases optical absorption in both long- and short-wavelength visible-range intervals.

The presented theoretical model, which assumes the presence of two types of deep defect centers so that each center is characterized by two states with different photoionization cross sections, satisfactorily describes the experimental dependences of the absorption coefficient in the BSO crystal on the radiation affecting the crystal.

This work was performed within the framework of the state order of the Ministry of Education and Science of the Russian Federation for 2013 (project No.7.2647.2011) with partial financial support of the Russian Foundation for Basic Research (project No. 12-02-90038-Bel_a) and the Belarusian Republican Foundation for Fundamental Research (project No. F12R-222).

\section{REFERENCES}

1. P. V. Dos Santos, J. Frejlich, and J. F. Carvalho, Appl. Phys. B, 81, 651 (2005).

2. M. P. Petrov, S. I. Stepanov, and A. V. Khomenko, Photorefractive Crystals in Coherent Optics [in Russian], Nauka, St.Petersburg (1992). 
3. V.K. Malinovsky, O. A. Gudaev, V. A. Gusev, et al., Photoinduced Phenomena in Sillenites [in Russian], Nauka, Moscow (1990).

4. A. L. Tolstik, A. Yu. Matusevich, M. G. Kisteneva, et al., Quant. Electron., 37, No. 11, 1027 (2007).

5. S. G. Odoulov, K. V. Shcherbin, A. N. Shumeljuk, J. Opt. Soc. Am. B, 11, 1780 (1994).

6. A. E. Mandel', A. M. Plesovskikh, S. M. Shandarov, et al., Rus. Phys. J., 46, No. 12, 1237 (2003).

7. S. V.Shlykov, V.I.Itkin, A.S.Akrestina, et al., in: Proc. XXVIth School on Coherent Optics and Holography, September 4-9, 200\%, Irkutsk, p. 398.

8. S. M. Shandarov, A. E. Mandel', M. G. Kisteneva, et al., High Energy Chem., 42, No. 7557 2008). 